\title{
Estratégias de enfrentamento: uma revisão sistemática sobre instrumentos de avaliação no contexto brasileiro
}

\section{Coping strategies: a systematic review on evaluation tools in the Brazilian context}

\section{Estrategias de enfrentamiento: una revisión sistemática sobre instrumentos de evaluación en el contexto brasilero}

\author{
Juceli Andrade Paiva Morero ${ }^{1}{ }^{(0,}$, Gabriela Rodrigues Bragagnollo2 ${ }^{2}$, \\ Maria Tereza Signorini Santos ${ }^{3}$ (])
}

\section{Histórico}

Recibido:

29 de enero de 2018

Aceptado:

10 de abril de 2018

1 Mestranda pela Escola de Enfermagem de Ribeirão Preto, Departamento de Enfermagem Psiquiátrica e Ciências Humanas. Universidade de São Paulo, São Paulo, SP, Brasil. Autor de Correspondência:

E-mail:juceli.morero@usp.br

2 Mestranda pela Escola de Enfermagem de Ribeirão Preto, Departamento de Saúde Pública. Universidade de São Paulo, São Paulo, SP, Brasil.

3 Mestranda pela Escola de Enfermagem de Ribeirão Preto, Departamento de Enfermagem Psiquiátrica e Ciências Humanas. Universidade de São Paulo, São Paulo, SP, Brasil.

Resumo

Introdução: $O$ enfrentamento caracteriza-se pela junção de estratégias cognitivas e comportamentais usadas pelos indivíduos, para o controle das demandas internas e externas e quando tais estratégias não são utilizadas de forma assertiva predispõem o indivíduo ao adoecimento. Este estudo teve como objetivo realizar uma revisão sistemática dos instrumentos para a avaliação das estratégias de enfrentamento adaptadas ao contexto brasileiro. Materiais e Métodos: Revisão sistemática da literatura realizada nas bases de dados EBSCO, MEDLINE/PubMed, Web of Science e Scopus, no período de 2002 - 2017. Resultados: Foram encontrados 305 artigos (português, espanhol e inglês), 33 artigos que tiveram critérios de elegibilidade e 11 artigos foram incluídos por tratarem de instrumentos validados que avaliam estratégias de enfrentamento. Sendo eles: Escala Modo de Enfrentamento de Problemas e Inventário de Estratégias de Coping. Discussão: Os instrumentos são compostos por adaptações transculturais, de estilo psicométrico e de autoaplicação. De modo geral, os instrumentos apresentaram boas evidências de validade e fidedignidade. As propriedades de validade e consistência interna (confiabilidade) dos instrumentos foram consideradas satisfatórias. Conclusões: Espera-se que esse trabalho incentive o avanço na busca de instrumentos de avaliação das estratégias de enfrentamento e que ofereça subsídios para o planejamento de intervenções mais assertivas, a fim de auxiliar os indivíduos no enfrentamento das situações conflitantes, minimizando o impacto do adoecimento.

Palavras chave: Adaptação Psicológica; Avaliação; Inquéritos e Questionários.

Introduction: Coping is characterized by the combination of cognitive and behavioral strategies used by individuals to control internal and external demands; when such strategies are not used assertively, they predispose the individual to illness. This study sought to conduct a systematic review of the instruments to evaluate coping strategies adapted to the Brazilian context. Materials and Methods: Systematic literature review carried out on the EBSCO, MEDLINE/ PubMed, Web of Science, and Scopus databases, during the period from 2002 to 2017. Results: The review found 305 articles (Portuguese, Spanish, and English), 33 articles fulfilled eligibility criteria and 11 articles were included dealing with validated instruments that evaluate coping strategies; with these instruments being: Scale on the Mode of Coping with Problems and Inventory of Coping Strategies. Discussion: The instruments comprised transcultural adaptations, psychometric style, and self-application. In general, the instruments presented good validity and reliability evidence. The validity and internal consistency (reliability) properties of the instruments were considered satisfactory. Conclusions: It is expected that this work will encourage progress in the search for tools to evaluate coping strategies and that it will provide aids in the planning of more assertive interventions to help individuals in coping with conflicting situations, minimizing the impact of illness.

Key words: Adaptation, Psychological; Evaluation; Surveys and Questionnaires.

Resumen

Introducción: El enfrentamiento se caracteriza por la unión de estrategias cognitivas y comportamentales usadas por los individuos para el control de las demandas internas y externas, y cuando tales estrategias no son utilizadas de forma asertiva predisponen al individuo a enfermarse. Este estudio tuvo como objetivo realizar una revisión sistemática de los instrumentos para la evaluación de las estrategias de enfrentamiento adaptadas al contexto brasilero. Materiales y Métodos: Revisión sistemática de la literatura realizada en las bases de datos EBSCO, MEDLINE/PubMed, Web of Science y Scopus en el período 2002-2017. Resultados: Se encontraron 305 artículos (portugués, español e inglés), 33 artículos tuvieron criterios de elegibilidad y 11 artículos se incluyeron por tratarse de instrumentos validados que evalúan estrategias de enfrentamiento. Siendo ellos: Escala Modo de Enfrentamiento de Problemas e Inventario de Estrategias de Coping. Discusión: Los instrumentos están compuestos por adaptaciones transculturales de estilo psicométrico y de autoaplicación. En general, los instrumentos presentaron buenas evidencias de validez y fidedignidad. Las propiedades de validez y consistencia interna (confiabilidad) de los instrumentos se consideraron satisfactorias. Conclusiones: Se espera que este trabajo incentive el avance en la búsqueda de instrumentos de evaluación de las estrategias de enfrentamiento y que ofrezca subvenciones para la planificación de intervenciones más asertivas, a fin de ayudar a los individuos en el enfrentamiento de las situaciones conflictivas, minimizando el impacto de la enfermedad.

Palabras clave: Adaptación Psicológica; Evaluación; Encuestas y Cuestionarios.

Como citar este artigo: Morero JAP, Bragagnollo GR, Santos MTS. Estratégias de enfrentamento: uma revisão sistemática sobre instrumentos de avaliação no contexto brasileiro. Rev Cuid. 2018; 9(2): 2257-68. http://dx.doi.org/10.15649/cuidarte.v9i2.503

(c) (1) (C)2018 Universidad de Santander. Este es un artículo de acceso abierto, distribuido bajo los términos de la licencia Creative Commons Attribution (CC BY-NC 4.0), que permite el uso ilimitado, distribución y reproducción en cualquier medio, siempre que el autor original y la fuente sean debidamente citados. 


\section{INTRODUÇÃO}

$\mathrm{O}$ enfrentamento caracteriza-se pela junção de estratégias cognitivas e comportamentais usadas pelos indivíduos, para o controle das demandas internas e externas, avaliadas como sobrecarga ou que excedam a capacidade do organismo. Segundo a teoria transacional ${ }^{1}$, o enfrentamento é um processo que decorre da interação entre o indivíduo e o ambiente, influenciado pelos traços de personalidade desta pessoa e experiências prévias que possa ter constituído para a aquisição de recursos emocionais, comportamentais, cognitivos e sociais para lidar com situações adversas ${ }^{1}$.

Tais estratégias de enfrentamento deverão acontecer, portanto no sentido de minimizar a pressão física, emocional e psicológica relacionada a acontecimentos desencadeantes de estresse, resultando no ajustamento psicossocial do indivíduo e consequentemente na melhoria da qualidade de vida e do equilíbrio mental ${ }^{2}$. Sendo assim, apenas esforços conscientes e intencionais são considerados estratégias de enfrentamento ${ }^{1,3}$.

De acordo com a literatura, são classificadas quanto a sua função, em duas categorias: a primeira categoria refere-se às estratégias focadas no problema e a segunda focada na emoção ${ }^{4}$ Na primeira o indivíduo busca conhecer o agente estressor e tenta de algum modo modificá-lo ou evitá-lo no futuro, já na segunda procura diminuir a aflição que o estímulo determina, mesmo que a situação estressante não possa ser modificada. Entre essas duas categorias existem uma inter-relação, que ocorre de forma dinâmica e recíproca, visto que, indivíduos expostos a uma mesma fonte geradora de estresse, poderão valer-se de ambas estratégias de enfrentamento. De modo que, uma estratégia que, previamente, estaria focada no problema, também poderia ter uma função focada na emoção ${ }^{5}$.

As estratégias de enfrentamento podem também ter impactos positivos ou negativos. Como exemplo, podemos citar uma pessoa em estado de ansiedade, que pode optar por fumar um cigarro ou utilizar técnicas de relaxamento. Ambas as estratégias reduzem o estado de ansiedade, no entanto, a última é considerada como estratégia mais saudável. Assim, as estratégias utilizadas pelo indivíduo tanto podem incluir respostas efetivamente positivas sobre o estressor como respostas negativas para a saúde e o bem-estar do mesmo $^{6,7}$.

Deste modo, ressalta-se que as estratégias de enfrentamento mostram-se como um importante ponto de empoderamento ou vulnerabilidade para um individua na superação de adversidades. O reconhecimento das estratégias de enfrentamento, nesta perspectiva, pode auxiliar o desenvolvimento de intervenções específicas para a promoção do bem-estar, qualidade de vida e minimizar o adoecimento ${ }^{8}$. Sendo esta uma importante área de intervenção para profissionais de saúde.

Diante o exposto, considerando que as estratégias de enfrentamento podem interferir no curso do adoecimento, é importante instrumentalizar os indivíduos para que possam lidar com situações estressoras, porém para ajudá-los a identificar e desenvolver estratégias mais adaptativas é imprescindível identificá-las. 
As estratégias de enfrentamento podem ser avaliadas através de entrevistas e análises qualitativas ou pelo uso de instrumentos psicrométricos e análises quantitativas. E diante da relevância do tema, este estudo teve como objetivo revisar e analisar quais são os instrumentos existentes para avaliação das estratégias de enfrentamento que sejam adaptadas para o contexto brasileiro?

\section{MATERIAIS E MÉTODOS}

Realizou-se uma revisão sistemática da literatura de acordo com os Principais Itens para Análises Sistemáticas e Meta-análises (PRISMA Statement $)^{9}$, com o objetivo de investigar quais os instrumentos existentes para a avaliação das estratégias de enfrentamento adaptadas ao contexto brasileiro.

Para a coleta dos dados, foi utilizado o acrônimo PICO:"P"- Population (especifica qual a população em foco); "I"- Intervention (define qual o tipo de intervenção estamos testando);“C" Comparison (identifica qual é o grupo controle que será testado juntamente com a intervenção) e “O” - Outcome (que são os desfechos que queremos avaliar) ${ }^{10}$.

O uso dessa estratégia para formular a questão de pesquisa na condução de métodos de revisão possibilita a identificação de palavras-chave, as quais auxiliam na localização de estudos primários relevantes nas bases de $\operatorname{dados}^{10}$. Assim, a questão de pesquisa delimitada foi: "Quais são as estratégias de enfrentamento encontradas na literatura que são adaptadas ao contexto brasileiro?" Nela, o primeiro elemento da estratégia $(\mathrm{P})$ consiste na população brasileira; o segundo (I), as estratégias; e o quarto elemento $(\mathrm{O})$, identificar os instrumentos existentes que são adaptados ao contexto brasileiro. Ressalta-se que, dependendo do método de revisão, não se emprega todos os elementos da estratégia PICO. Nesta revisão, o terceiro elemento, ou seja, a comparação, não foi utilizada.

A busca de dados foi realizada de forma duplo cega independente, no período de abril a junho de 2017 (Morero JAP, Bragagnollo GR, Santos MTS). Foram inclusos artigos originais em português, inglês e espanhol, que utilizaram um instrumento de coleta de dados para avaliar a estratégias de enfrentamento adaptadas ao contexto brasileiro, disponíveis na integra, nos últimos 15 anos (2002 - 2017), conforme encontrado nos descritores de Ciências da Saúde (DeCS), disponíveis nas bases de dados: EBSCO, MEDLINE/PubMed, Web of Science e Scopus, foram excluídos estudos dos quais não estavam disponíveis na íntegra; outras revisões da literatura e estudos cujo foco principal foi a avaliação de outros construtos que não estratégias de enfrentamento. Para as bases EBSCO, Web of Science e Scopus, foram utilizados os seguintes termos de busca com operadores booleanos: "(adaptation, psychological) AND (translating) AND (studies) AND (instruments OR test OR questionnaires OR checklist ) AND (Brazil)". Para a base MEDLINE/PubMed, foram utilizados os seguintes termos "(adaptation, psychological) AND (studies OR evaluation studies) AND (translating) AND (instrument OR scale OR test OR inventory OR interview OR questionnaire OR checklist ORscreen)AND (BrazilOR Brazilian OR Portuguese)". Todos os artigos foram inclusos no gerenciador de arquivos Zotero (disponível em: https:/www.zotero.org/ ), assim foi possível resolver todos as duplicatas pela autora Santos MTS. 
Avaliação da qualidade metodológica dos list do instrumento (desde que o critério fosse estudos pertinente ao desenho) entraram na revisão.

Foi realizada a inclusão dos artigos de acordo com

RESULTADOS

os Principais Itens para Análises Sistemáticas e

Meta-análises (PRISMA Statement) ${ }^{9}$. Estudos

A Figura1, mostra o Fluxograma utilizado para a com pelo menos 7 itens atendidos no check seleção dos estudos (PRISMA).

Registros identificados através da pesquisa de base de dados $(n=305)$

EBSCO 52, PubMed 156, Web of Science 64, Scopus 33
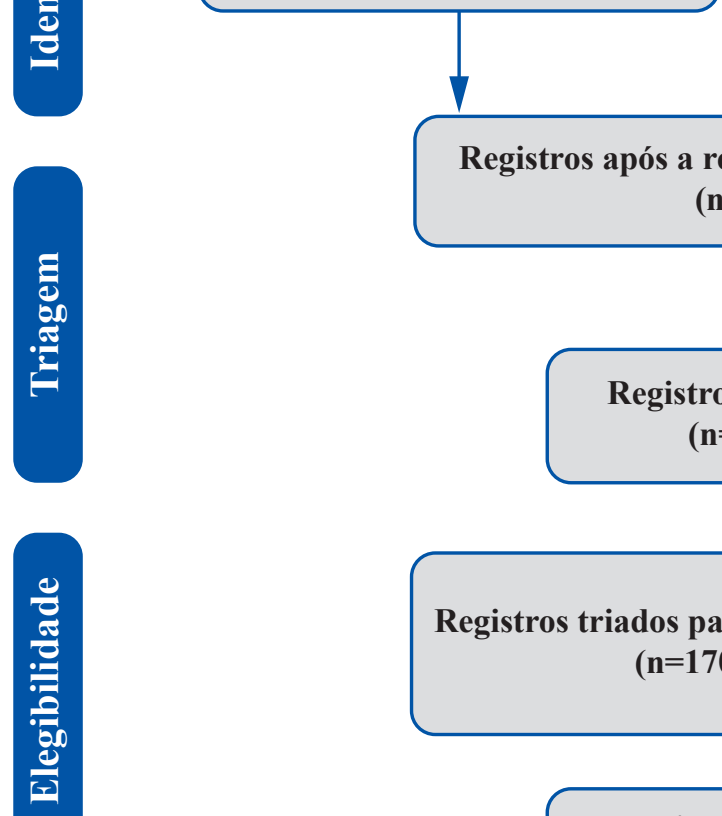

Registros após a remoção de duplicatas $(n=37)$
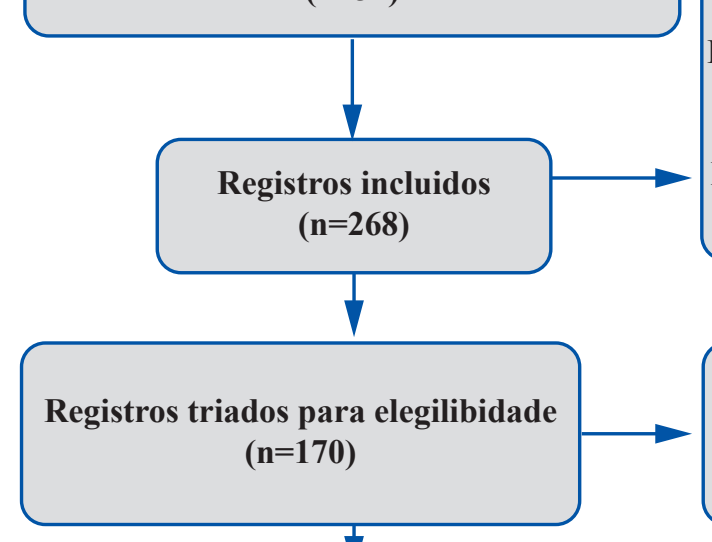

Estudios incluidos na sintese qualitativa $(n=33)$

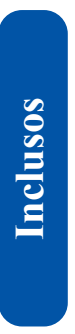

Registros excluidos baseados nos critérios de Inclusão e exclusão

$(n=137)$

Registros excluidos com base no título $(n=98)$

Sem relevância $(n=21)$

Estudo diferente do estudo da revisão

\section{Registros excluidos} $(n=22)$

Nenhum resultado relevante $(n=22)$
Estudios incluidos na sintese qualitativa $(\mathbf{n}=11)$

EBSCO 2, PubMed 6, Web of Science 3, Scopus 0

Figura 1. Fluxograma utilizado para a seleção dos estudos (PRISMA) 
Nesta revisão, foram incluídos 11 artigos originais que atenderam aos critérios de inclusão previamente estabelecidos e, a seguir, apresentarse-á um panorama geral dos artigos avaliados. As referências completas destes estão destacadas com asterisco $(*)$ na lista de referências deste artigo.

Entre os artigos incluídos, oito são de autoria de psicólogos, um tem entre seus autores um médico e uma psicóloga, um foi redigido por fisioterapeutas e terapeutas ocupacionais e um foi redigido apenas por médicos.

Em relação ao local do estudo, sete foram desenvolvidos em estabelecimentos hospitalares, um em uma clínica privada e dois em universidades. Constatou- se, também, que uma pesquisa foi realizada em um Centro de Referência à Saúde do Trabalhador.
Quanto aos tipos de periódicos nos quais foram publicados os artigos avaliados, quatro foram publicados em periódicos de psicologia, dois em periódicos de psiquiatria, um em periódico médico, um em periódico de enfermagem e três foram publicados em periódicos de outras áreas da saúde. Quanto ao ano de publicação, em 2004, 2007, 2011 e 2012 foram respectivamente um e cinco no ano de 2015.

Quanto ao tipo de delineamento de pesquisa dos artigos avaliados, evidenciou-se, na amostra: seis estudos com delineamento transversal, um ensaio clínico randomizado controlado, dois ensaios clínicos controlado, um estudo de coorte e um estudo com delineamento de pesquisa experimental.

Na Tabela 1, apresenta-se a síntese dos artigos incluídos na presente revisão.

Tabela 1. Síntese dos artigos incluídos

\begin{tabular}{|c|c|c|c|c|c|}
\hline $\begin{array}{l}\text { Título do } \\
\text { artigo }\end{array}$ & $\begin{array}{l}\text { Autores } \\
\text { e Ano }\end{array}$ & Objetivo & $\begin{array}{c}\text { Instrumentos de } \\
\text { enfrentamento }\end{array}$ & $\begin{array}{l}\text { Principais } \\
\text { resultados }\end{array}$ & Conclusões \\
\hline $\begin{array}{l}\text { Adaptação } \\
\text { acadêmica e } \\
\text { Coping em } \\
\text { estudantes } \\
\text { universitários }^{11}\end{array}$ & $\begin{array}{l}\text { Carlotto } \\
\text { et al. } \\
2015\end{array}$ & $\begin{array}{l}\text { Investigar a relação } \\
\text { entre estratégias de } \\
\text { coping e a adaptação } \\
\text { acadêmica em } \\
\text { universitários }\end{array}$ & $\begin{array}{c}\text { Escala Modo de } \\
\text { Enfrentamento } \\
\text { de Problemas } \\
\text { (EMEP) }\end{array}$ & $\begin{array}{l}\text { Em relação ao gênero, as } \\
\text { mulheres apresentaram } \\
\text { maiores estratégias focadas } \\
\text { na emoção, práticas } \\
\text { religiosas e suporte social. } \\
\text { Não houve diferença no uso } \\
\text { de estratégias entre os } \\
\text { estudantes. }\end{array}$ & $\begin{array}{l}\text { As estratégias focadas no } \\
\text { problema e no suporte social } \\
\text { correlacionaram positivamente } \\
\text { com a adaptação acadêmica. } \\
\text { Em contrapartida, a emoção e } \\
\text { as práticas religiosas se } \\
\text { relacionaram negativamente } \\
\text { diferença no uso de estratégias } \\
\text { entre os estudantes. }\end{array}$ \\
\hline $\begin{array}{l}\text { Estratégias de } \\
\text { enfrentamento } \\
\text { adotadas por } \\
\text { pacientes com } \\
\text { doença renal } \\
\text { crônica em } \\
\text { preparação } \\
\text { para o transplan- } \\
\text { te renal }{ }^{12}\end{array}$ & $\begin{array}{l}\text { Kohlsdorf } \\
\text { et } \text { al. } \\
2015\end{array}$ & $\begin{array}{l}\text { Analisar as estraté- } \\
\text { gias de enfrentamen- } \\
\text { to adotadas por } \\
\text { pacientes em } \\
\text { preparação para } \\
\text { transplante renal }\end{array}$ & $\begin{array}{c}\text { Escala Modo de } \\
\text { Enfrentamento } \\
\text { de Problemas } \\
\text { (EMEP) }\end{array}$ & $\begin{array}{l}\text { As principais estratégias de } \\
\text { enfrentamento utilizadas } \\
\text { foram: práticas religiosas, } \\
\text { enfrentamento focado no } \\
\text { problema e suporte social. }\end{array}$ & $\begin{array}{c}\text { Os resultados também } \\
\text { apontam associação entre } \\
\text { algum dado sócio demográfico } \\
\text { com as estratégias de } \\
\text { enfrentamento. }\end{array}$ \\
\hline
\end{tabular}




\section{Tabela 1. (continuação)}

\begin{tabular}{|c|c|c|c|c|c|}
\hline $\begin{array}{c}\text { Sessões de } \\
\text { reforço após } \\
\text { Grupo de } \\
\text { Terapia } \\
\text { Cognitivo } \\
\text { Comportamental } \\
\text { (TCC) para o } \\
\text { Transtorno } \\
\text { do Pânico: } \\
\text { impacto na } \\
\text { resiliência, } \\
\text { Coping, e } \\
\text { qualidade de } \\
\text { vida }^{13} \\
\end{array}$ & $\begin{array}{l}\text { Wesner } \\
\text { et al. } \\
2015\end{array}$ & $\begin{array}{l}\text { Avaliar o impacto } \\
\text { do reforço após } \\
\text { sessões de técnicas } \\
\text { cognitivas sobre } \\
\text { estratégias de } \\
\text { enfrentamento, } \\
\text { resiliência e } \\
\text { qualidade de vida } \\
\text { em pacientes } \\
\text { submetidos à TCC. }\end{array}$ & $\begin{array}{l}\text { Inventário de } \\
\text { Estratégias de } \\
\text { coping }\end{array}$ & \begin{tabular}{|c|} 
Observou-se um aumento no \\
domínio das relações sociais \\
no grupo de reforço \\
Enfrentamento e outros \\
domínios de qualidade de \\
vida não se alteraram após as \\
sessões. Alterações na \\
resiliência foram dependen- \\
tes da intensidade dos \\
sintomas.
\end{tabular} & $\begin{array}{l}\text { Os resultados positivos } \\
\text { causados pelas sessões talvez } \\
\text { possam ser um resultado do } \\
\text { formato do grupo de } \\
\text { intervenção. } \\
\text { Sendo estas sessões de reforço } \\
\text { úteis para manter os benefícios } \\
\text { obtidos com a TCC. }\end{array}$ \\
\hline $\begin{array}{l}\text { Enfrentamento, } \\
\text { psicopatologia, e } \\
\text { qualidade de vida } \\
\text { em pacientes } \\
\text { com câncer sob } \\
\text { cuidados } \\
\text { paliativos }^{14} \text {. }\end{array}$ & $\begin{array}{l}\text { Sorato } \\
\text { et al. } \\
2015\end{array}$ & $\begin{array}{l}\text { Avaliar desespe- } \\
\text { rança, ansiedade, } \\
\text { depressão } \\
\text { e qualidade de vida } \\
\text { em pacientes com } \\
\text { câncer submetido a } \\
\text { tratamento paliativo, } \\
\text { avaliando possíveis } \\
\text { correlações com } \\
\text { estratégias de } \\
\text { enfrentamento. }\end{array}$ & $\begin{array}{l}\text { Inventário de } \\
\text { Estratégias de } \\
\text { coping }\end{array}$ & \begin{tabular}{|c} 
o confronto, busca de apoio \\
social e reavaliação positiva \\
foram inversamente \\
correlacionadas com \\
desesperança. Busca de apoio \\
social, resolução de \\
problemas, e \\
reavaliação positiva foram \\
inversamente correlacionadas \\
com indicadores de \\
depressão. Em contraste, o \\
uso de a estratégia de fuga e \\
esquiva e a utilização \\
reduzida da estratégia de \\
resolução de problemas \\
foram associada com o \\
aumento da ansiedade.
\end{tabular} & $\begin{array}{l}\text { A estratégia de enfrentamento } \\
\text { focado no problema exerceu } \\
\text { impacto positivo sobre o } \\
\text { processo de fim de vida, } \\
\text { permitindo-lhes procurar } \\
\text { alternativas para experimentar } \\
\text { esse processo de uma forma } \\
\text { mais ajustada. }\end{array}$ \\
\hline $\begin{array}{l}\text { Efeito do grupo } \\
\text { de terapia } \\
\text { cognitivo-com- } \\
\text { portamental } \\
\text { (TCC) para } \\
\text { transtorno do } \\
\text { pânico (TP) na } \\
\text { mudança das } \\
\text { estratégias de } \\
\text { enfrentamento }\end{array}$ & $\begin{array}{l}\text { Wesner } \\
\text { et al. } \\
2014\end{array}$ & $\begin{array}{c}\text { avaliar o efeito da } \\
\text { TCC na escolha da } \\
\text { estratégia de } \\
\text { enfrentamento em } \\
\text { pacientes com (TP) } \\
\text { comparado com um } \\
\text { grupo de indivíduos } \\
\text { sem transtornos } \\
\text { mentais }\end{array}$ & $\begin{array}{l}\text { Inventário de } \\
\text { Estratégias de } \\
\text { coping }\end{array}$ & $\begin{array}{c}\text { A TCC foi eficaz na redução } \\
\text { da gravidade em pacientes } \\
\text { com TP. Estes utilizaram um } \\
\text { número menor de estratégias } \\
\text { de confronto e escape após a } \\
\text { TCC }\end{array}$ & $\begin{array}{c}\text { Apesar de as alterações } \\
\text { observadas após a TCC, a } \\
\text { escolha da estratégia de } \\
\text { enfrentamento ainda era } \\
\text { diferente do grupo controle. } \\
\text { Outras ferramentas cognitivas } \\
\text { devem ser incluídas para tratar } \\
\text { estratégias mal adaptativas de } \\
\text { enfrentamento. }\end{array}$ \\
\hline $\begin{array}{l}\text { Estilo de coping e } \\
\text { sintomatologia } \\
\text { depressiva } \\
\text { durante a } \\
\text { gravidez de uma } \\
\text { amostra em um } \\
\text { ambiente } \\
\text { privado }^{16}\end{array}$ & $\begin{array}{l}\text { Faisal- } \\
\text { Cury } \\
\text { et } \text { al. } \\
2012\end{array}$ & $\begin{array}{c}\text { avaliar a associação } \\
\text { da sintomatologia } \\
\text { depressiva (SD) } \\
\text { pré-natal com } \\
\text { eventos de vida e } \\
\text { estilos de } \\
\text { enfrentamento }\end{array}$ & $\begin{array}{l}\text { Inventário de } \\
\text { Estratégias de } \\
\text { Coping }\end{array}$ & $\begin{array}{l}\text { Todos os estilos de } \\
\text { enfrentamento foram } \\
\text { associados com SD, exceto } \\
\text { em busca de apoio e } \\
\text { reavaliação positiva. }\end{array}$ & $\begin{array}{l}\text { SD foi altamente prevalente e } \\
\text { foi associado com estilos de } \\
\text { enfrentamento mal adaptativos }\end{array}$ \\
\hline $\begin{array}{c}\text { Impacto do } \\
\text { manejo clínico } \\
\text { da dor: avaliação } \\
\text { de estresse e } \\
\text { enfrentamento } \\
\text { entre } \\
\text { profissionais } \\
\text { de saúde } \\
\end{array}$ & $\begin{array}{l}\text { Negro- } \\
\text { monte } \\
\text { et al. } \\
2011\end{array}$ & \begin{tabular}{|c|} 
investigar a \\
percepção de estresse \\
e as estratégias de \\
enfrentamento de 31 \\
profissionais de \\
diferentes categorias.
\end{tabular} & $\begin{array}{l}\text { Inventário de } \\
\text { Estratégias de } \\
\text { Coping }\end{array}$ & $\begin{array}{c}\text { Comparativamente às demais } \\
\text { categorias, os técnicos de } \\
\text { enfermagem indicaram } \\
\text { mais fatores de estresse. Em } \\
\text { compensação, informaram } \\
\text { maior diversidade de } \\
\text { estratégias de enfrentamento. }\end{array}$ & $\begin{array}{c}\text { também revelam } \\
\text { disponibilidade de fatores de } \\
\text { proteção, apontando } \\
\text { perspectivas de intervenção } \\
\text { preventiva, destinada à equipe } \\
\text { de enfermagem }\end{array}$ \\
\hline $\begin{array}{c}\text { Estratégias de } \\
\text { enfrentamento } \\
\text { da fissura em } \\
\text { dependentes de } \\
\text { nicotina }^{18}\end{array}$ & $\begin{array}{l}\text { Araujo } \\
\text { et al. } \\
2009\end{array}$ & $\begin{array}{c}\text { investigar a } \\
\text { associação entre a } \\
\text { intensidade da } \\
\text { fissura e os tipos de } \\
\text { coping utilizados por } \\
\text { dependentes de } \\
\text { tabaco }\end{array}$ & $\begin{array}{l}\text { Inventário de } \\
\text { Estratégias de } \\
\text { Coping }\end{array}$ & $\begin{array}{l}\text { As estratégias de coping } \\
\text { mais utilizadas, em ordem } \\
\text { decrescente, foram } \\
\text { aceitação de responsabili- } \\
\text { dade, autocontrole e } \\
\text { distanciamento }\end{array}$ & $\begin{array}{l}\text { A fissura mais intensa pode } \\
\text { levar o dependente a utilizar } \\
\text { estratégias de confrontamento } \\
\text { que são ineficientes } \\
\text { na contenção das emoções e } \\
\text { no enfrentamento das situações } \\
\text { de risco de recaída }\end{array}$ \\
\hline
\end{tabular}


Tabela 1. (continuação)

\begin{tabular}{|c|c|c|c|c|c|}
\hline $\begin{array}{l}\text { Satisfação de } \\
\text { Vida dos } \\
\text { Trabalhadores } \\
\text { com relação a } \\
\text { Distúrbios } \\
\text { Osteomusculares } \\
\text { causados pelo } \\
\text { trabalho (DOCT) } \\
\text { no Brasil: } \\
\text { associações } \\
\text { com sintomas, } \\
\text { limitação } \\
\text { funcional e } \\
\text { enfrentamento }\end{array}$ & $\begin{array}{l}\text { Viana } \\
\text { et al. } \\
2007\end{array}$ & $\begin{array}{c}\text { analisar a } \\
\text { contribuição de } \\
\text { diversas variáveis } \\
\text { em direção a } \\
\text { satisfação com a } \\
\text { vida entre os } \\
\text { trabalhadores com } \\
\text { Distúrbios } \\
\text { Osteomusculares } \\
\text { Relacionados ao } \\
\text { trabalho. }\end{array}$ & $\begin{array}{l}\text { Inventário de } \\
\text { Estratégias de } \\
\text { Coping }\end{array}$ & $\begin{array}{l}\text { Os trabalhadores relataram } \\
\text { maiores níveis de estratégias } \\
\text { focados na emoção e } \\
\text { menores níveis de } \\
\text { enfrentamento focados } \\
\text { no problema. }\end{array}$ & $\begin{array}{l}\text { Intervenções dirigidas aos } \\
\text { trabalhadores com DOCT que } \\
\text { procuram promover o } \\
\text { bem-estar devem favorecer o } \\
\text { enfrentamento da forma mais } \\
\text { adaptativa. }\end{array}$ \\
\hline $\begin{array}{c}\text { Eventos } \\
\text { adversos da vida } \\
\text { e habilidades de } \\
\text { enfrentamento } \\
\text { no Transtorno de } \\
\text { Pânico (TP) }\end{array}$ & $\begin{array}{l}\text { Savoia } \\
\text { et al. } \\
2004\end{array}$ & $\begin{array}{l}\text { investigar a relação } \\
\text { entre eventos de } \\
\text { vida, habilidades de } \\
\text { enfrentamento e } \\
\text { transtorno do pânico. }\end{array}$ & $\begin{array}{l}\text { Inventário de } \\
\text { Estratégias de } \\
\text { Coping }\end{array}$ & $\begin{array}{c}\text { pacientes com TP em } \\
\text { comparação com controles } \\
\text { relataram perda de apoio } \\
\text { social e em situações de } \\
\text { estresse, tem usado } \\
\text { frequentemente habilidades } \\
\text { de enfrentamento julgadas } \\
\text { como ineficazes }\end{array}$ & $\begin{array}{l}\text { Os resultados sugerem que o } \\
\text { tipo de evento de vida e as } \\
\text { habilidades de enfrentamento } \\
\text { utilizado em resposta a eles, } \\
\text { mais do que a ocorrência de } \\
\text { eventos de estresse em si, pode } \\
\text { ser associado com o } \\
\text { aparecimento do TP }\end{array}$ \\
\hline $\begin{array}{l}\text { Estresse e coping } \\
\text { em uma amostra } \\
\text { de estudantes de } \\
\text { medicina no } \\
\text { Brasil }^{21}\end{array}$ & $\begin{array}{c}\text { Bassols } \\
\text { et al. } \\
2016\end{array}$ & $\begin{array}{l}\text { avaliar a prevalência } \\
\text { de estresse e } \\
\text { enfrentamento em } \\
\text { estudantes de uma } \\
\text { escola médica } \\
\text { pública no Brasil, } \\
\text { comparando-se os } \\
\text { grupos a partir do } \\
\text { primeiro e sexto } \\
\text { anos de treinamento }\end{array}$ & $\begin{array}{l}\text { Inventário de } \\
\text { Estratégias de } \\
\text { Coping }\end{array}$ & $\begin{array}{c}\text { sintomas de estresse foram } \\
\text { maiores em estudantes do } \\
\text { primeiro ano. Variáveis } \\
\text { associadas ao estresse foram: } \\
\text { ano de formação, renda, } \\
\text { satisfação com a formação e } \\
\text { o uso de estratégia de fuga / } \\
\text { esquiva. }\end{array}$ & $\begin{array}{c}\text { Considerando-se os sintomas } \\
\text { de estresse mais elevados entre } \\
\text { os estudantes do primeiro ano } \\
\text { de medicina e a associação } \\
\text { positiva da estratégia de fuga / } \\
\text { esquiva. } \\
\text { Assim, é importante desenvol- } \\
\text { ver estratégias mais adaptati- } \\
\text { vas para capacitar os alunos } \\
\text { para melhor enfrentamento das } \\
\text { situações de estresse. }\end{array}$ \\
\hline
\end{tabular}

*Elaborado pelos autores

Na Tabela 2, estão expostos os resultados obtidos na avaliação da consistência interna dos instrumentos referentes às estratégias de enfrentamento encontradas neste estudo.

Tabela 2. Resultados da consistência interna dos instrumentos encontrados no estudo

\begin{tabular}{|c|c|}
$\begin{array}{c}\text { Instrumentos de } \\
\text { enfrentamento }\end{array}$ & $\begin{array}{c}\text { Coeficiente Alfa } \\
\text { de Cronbach }\end{array}$ \\
\hline EMEP* & 0,74 a 0,84 \\
\hline IEC $* *^{*}$ & 0,81 a 0,84 \\
\hline
\end{tabular}

*EMEP (Escala Modo de Enfrentamento de Problemas); **IEC (Inventário de Estratégias de Coping) Elaborado pelos autores.
De modo geral, os resultados são positivos, com coeficientes em um alcance satisfatório. Porém, nem todas as variáveis tiveram um valor de alfa aceitável, pois há alguns valores indicadores de relação entre itens com tendência moderada. Assim, usualmente são preferidos valores de alfa entre 0,80 e $0,90^{22}$, como resultado de alta confiabilidade.

\section{DISCUSSÃO}

O modo como os indivíduos gerenciam as situações estressoras da vida tem sido foco 
de averiguação e estudos ${ }^{23,24}$, com o intuito de buscar formas de auxiliar os indivíduos no manejo de eventos e agravos decorrentes de tais ocasiões que impactam no adoecimento. $\mathrm{Na}$ presente revisão, observou-se que atualmente no Brasil, ainda há uma escassez de instrumentos disponíveis para avaliação das estratégias de enfrentamento. Foram encontrados apenas dois instrumentos validados para utilização dentro do contexto brasileiro, que serão elencados a seguir, com ênfase em suas principais características e seus processos de validação.

O primeiro instrumento encontrado foi a "Escala Modo de Enfrentamento de Problemas" (EMEP) construída em 1985 por Vitaliano et $\mathrm{al}^{25}$, foi idealizada com base no modelo interacional do estresse, que permite mensurar estratégias de enfrentamento em relação a estressores específicos ${ }^{26}$. A versão original é composta por 57 itens, distribuídos em 5 fatores. Adaptada ao contexto brasileiro por Gimenes e Queiroz ${ }^{27}$, e validada por Seidl, Tróccoli e Zannon ${ }^{26}$. A escala validada é composta por 45 itens, distribuídos em 4 fatores: fator 1 - enfrentamento focalizado no problema (18 itens, $\alpha=0,84)$; fator 2enfrentamento focalizado na emoção (15 itens, $\alpha=0,81$ ); fator 3 - busca de suporte social ( 5 itens, $\alpha=0,70$; e fator 4 - busca de práticas religiosas (7 itens, $\alpha=0,74$ ). As alternativas de respostas estão dispostas em escala do tipo Likert de cinco pontos, contendo os seguintes intervalos: 1- Eu nunca faço isso; 2- Eu faço isso um pouco; 3 - Eu faço isso às vezes; 4- Eu faço isso muito; 5- Eu faço isso sempre. Deste modo, o escore total é obtido pela média aritmética e, quanto mais alto o escore, maior a frequência de utilização da estratégia de enfrentamento. Quanto aos termos de fidedignidade e validade, este instrumento possui boas qualidades psicométricas. A validade de construto foi concretizada através da analise da estrutura fatorial, pelo Método dos Fatores Principais, rotação ortogonal Varimax. O valor mínimo de 0,35 foi estabelecido o para a carga fatorial. A análise fatorial dos quatro fatores explicou $25,7 \%$ da variância total, sendo que o fator um explicou 9,2\%, o fator dois 7,5\%, o fator três $5 \%$ e o fator quatro $3,9 \%$. Para avaliação da consistência interna dos fatores utilizou-se o alfa de Cronbach que é uma ferramenta estatística que quantifica, numa escala de 0 a 1 , a confiabilidade de um questionário, sendo o valor mínimo aceitável para se considerar um questionário confiável é 0,7. Na EMEP, foram encontrados os seguintes valores de alfa de Cronbach: 0,84 no fator $1 ; 0,81$ no fator $2 ; 0,70$ no fator 3 ; e 0,74 no ultimo fator. As subescalas são avaliadas separadamente, não havendo um escore global ${ }^{26}$.

O segundo instrumento localizado foi $\mathrm{o}$ "Inventário de Estratégias de Coping"(IEC) ${ }^{28}$, adaptado para o Brasil por Savóia et $\mathrm{al}^{29}$, que investiga as estratégias de enfrentamento utilizadas pelos participantes em uma situação de desconforto com o próprio corpo. Cada aplicação do inventário centraliza-se no processo de coping de uma situação particular e não no coping como estilos ou traços de personalidade. A situação é que determina o padrão de estratégias de coping, e não variáveis pessoais. O Inventário é composto por 66 itens, em que a resposta a cada item é registrada a partir de uma escala Likert de zero (“ não usei esta estratégia”) a 3 ("usei em grande quantidade"). As estratégias 
de enfrentamento são: confronto, afastamento, autocontrole, suporte social, aceitação de responsabilidade, fuga e esquiva, resolução de problemas e reavaliação positiva. A escala não apresenta pontuação total como somatória para avaliação, sendo que os itens devem ser avaliados por meio dos escores médios dentro de cada estratégia ${ }^{30}$. Portanto, para a análise dos dados, são somados os escores atribuídos a cada item de um mesmo fator e divididos pelo número total de itens, identificando assim os fatores de coping utilizados pelos indivíduos para enfrentar o estresse. As correlações obtidas na validação para o Brasil apresentaram valores de 0,424 a $0,688^{29}$, O valor do coeficiente alfa de Cronbach foi entre 0,81 a 0,84, o que indica uma boa homogeneidade e consistência dos itens. Os autores também propuseram a divisão dos fatores de coping em outras duas subescalas, da seguinte forma: enfrentamento focado no problema (confronto e resolução de problemas) e enfrentamento focado na emoção (afastamento, autocontrole, aceitação de responsabilidade, reavaliação positiva, fuga e esquiva). $\mathrm{O}$ fator suporte social engloba ambos ${ }^{30}$.

Ambos os instrumentos foram utilizados para avaliar as estratégias de enfrentamento com grau de profundidade bastante variado, voltado para um perfil heterogêneo (estudantes, pacientes psiquiátricos, pacientes em tratamento dialítico, profissionais da saúde, entre outros).

Destaca-se que enquanto a Escala Modo de Enfrentamento de Problemas, de Vitaliano et al. (1985), agrupa as estratégias de enfrentamento em por meio quatro fatores (enfrentamento focalizado no problema, enfrentamento focalizado na emoção, busca de suporte social e busca de práticas religiosas), o Inventário de Estratégias de Coping, de Folkman et al. (1985) agrupa as estratégias de enfrentamento em uma maior diversidade de tipos de resultados (confronto, afastamento, autocontrole, suporte social, aceitação de responsabilidade, fuga e esquiva, resolução de problemas e reavaliação positiva), o que permite uma análise mais abrangente sobre as diversas possibilidades de comportamentos para lidar com estressores.

Entre os estudos de associação, a estratégia de enfrentamento é, em sua maioria, relacionada a intervenção como a Terapia Cognitiva Comportamental - TCC ( $\mathrm{n}=3 ; 28 \%)$, seguido de bem-estar no ambiente laboral $(\mathrm{n}=2 ; 18 \%)$, estresse entre estudantes $(n=2 ; 18 \%)$, pacientes em tratamento médico $(n=2 ; 18 \%)$ e Depressão e Transtorno do Pânico (cada um com um artigo; 18\%). Nos estudos sobre TCC, o constructo tem sido elemento de grande interesse, especialmente em como o uso de determinadas estratégias pode influenciar no bem-estar dos indivíduos em uso dessa terapia.

Outroponto importanteéque entre os instrumentos para avaliação de estratégias de enfrentamento no contexto brasileiro apresentados nesta revisão, conforme citados acima, destaca-se o estudo a partir do modelo de Folkman e Lazarus (1985), considerando ser destes autores o instrumento mais utilizado nos achados da presente revisão ( $\mathrm{n}=982 \%$ ), na sequência a Escala Modo de enfrentamento de problemas $(n=2 ; 28 \%)$. 
Entre as estratégias mais utilizadas nos estudos, observou-se que a grande parte dos artigos apontaram a estratégia de resolução de problemas como a mais utilizada, sendo a de confronto como a menos utilizada. A estratégia de resolução de problemas implica um plano adequado para lidar com os diferentes estressores, em que, ao invés de esquivar-se da situação estressante, o indivíduo busca a solução, modificando suas atitudes, assim, está apto para gerenciar as fontes estressoras e, com isso, reduz ou elimina seu impacto.

Quanto aos estudos com intervenção $(n=3 ; 28 \%)$, observou-se que houve alteração na estratégia de enfrentamento utilizada pelos participantes antes e após a Terapia Cognitiva Comportamental. Os resultados mostraram houve aumento no uso de estratégias de enfrentamento focadas no problema, tais como controle e resolução de problemas. Dados estes apontam que o uso das intervenções são essenciais para que os indivíduos possam desenvolver capacidade de enfrentamento eficazes de acordo com cada situação vivenciada, refletindo no aumento do grau de funcionalidade tanto individual, quanto nas familias ${ }^{31}$.

Em relação as limitações desta revisão, é necessário enfatizar a necessidade de balancear as amostras em relação ao sexo, pois houve um número maior de mulheres em relação aos homens na composição das amostras. Também foram encontradas grande variabilidade no tamanho amostral, idade, perfil e ausência de análise mais apurada na maioria dos estudos expostos. Outra limitação importante, está relacionada com a carência de estudos com o mesmo objetivo deste, pois há uma escassez de artigos originais sobre o tema, o que impossibilita o enriquecimento da dados, exemplos e vivências, essenciais para a melhoria da assistência e redução do adoecimento. Por fim, outra limitação encontrada foi a grande variabilidade e diversos problemas metodológicos (ausência de grupo controle, análise de domínio de observação do estudo, cálculo amostral e a utilização do check list PRISMA que é utilizado para escrever de maneira adequada os resultados de uma revisão sistemática, porém existem outros check lists que também poderiam ter sido utilizados).

Entre as contribuições desta revisão, pode-se dizer que é um estudo inovador por não existir nenhum outro, seja na literatura nacional como também na internacional, com as mesmas características. Assim, os resultados revelam dados relevantes no contexto dos instrumentos para avaliar as estratégias de enfrentamento no Brasil.

\section{CONCLUSÕES}

Este estudo possibilitou a identificação de instrumentos utilizados para avaliação das estratégias enfrentamento que estão adaptados para o contexto brasileiro e que tem sido, frequentemente, utilizado nas pesquisas. Os instrumentos são compostos por adaptações transculturais, de estilo psicométrico e de autoaplicação, apresentando boas evidências de validade e fidedignidade. 
Observou-se também uma escassez de instrumentos disponíveis para tal, no Brasil. $\mathrm{O}$ que evidencia a necessidade de ampliar estudos e de construir instrumentos, a fim de, testar as proposições teóricas produzidas no contexto brasileiro, bem como, contribuir para o progresso do conhecimento científico nesta area. O desenvolvimento de instrumentos de avaliação das estratégias de enfrentamento oferece subsídios para o planejamento de intervenções mais assertivas, a fim de auxiliar os indivíduos no enfrentamento das situações conflitantes, minimizando o impacto do adoecimento.

Por fim, também se faz necessário que os profissionais e pesquisadores obtenham conhecimento acerca das ferramentas de avaliação existentes, pois estas oferecem subsídios para o planejamento de intervenções mais assertivas, a fim de auxiliar os indivíduos a lidar diante das dificuldades e no aprendizado de quais estratégias podem ajudá-los a atingir seus objetivos em diversas situações conflitantes, contribuindo assim para minimizar o impacto do adoecimento. Cabendo aos mesmos definir qual é a que melhor se aplica à sua realidade de atuação e objetivos.

Conflito de interesses: Os autores declaram não ter nenhum conflito de interesses.

\section{REFERÊNCIAS}

1. Lazarus R, Folkman S. Stress, appraisal, and coping. New York: Springer. 1984; 6-8.

2. Pereira TB, Rodrigues VLB. As estratégias de coping na promoção à saúde mental de pacientes oncológicos: uma revisão bibliográfica. Rev. Psicol. Saúde. 2016; 8 (1): 2431. http://dx.doi.org/10.20435/2177093X2016104

3. Mussumeci AM, Ponciano TEL. Estresse, coping e experiências emocionais: uma análise das respostas de enfrentamento do casal. Pensando famílias. 2017; 21(1): 33-49.
4. Siqueira DS, Figueiredo AEPL, Costa BEP, Riegel F. Perfil de enfrentamento (coping) e qualidade de vida dos pacientes pré e pós-transplante renal. Rev enferm UFPE on line. 2016; 10(1): 371-4.

5. Trentini M, Silva SH, Valle ML, Hammers SKSA. Enfrentamento de situações adversas e favoráveis por pessoas idosas em condições crônicas de saúde. Rev Latino-Am Enfermagem. 2005; 13(1): 38-45. http://dx.doi.org/10.1590/S0104-11692005000100007

6. Umann J, Silva M, Benavente SBT, Guido LA. O impacto das estratégias de enfrentamento na intensidade de estresse de enfermeiras de hemato-oncologia. Rev. Gaúcha Enferm. 2014; 35(3): 103-10.

http://dx.doi.org/10.1590/1983-1447.2014.03.44642

7. Kleinubing RE, Goulart CT, Silva RM, Umann J, Guido LA. Estresse e coping em enfermeiros de terapia intensiva adulto e cardiológica. Revista de Enfermagem da UFSM. 2013; 3(2): 335-44 https://doi.org/10.5902/217976928924

8. Ribeiro RM, Pompeo DA, Pinto MH, Ribeiro RC. Estratégias de enfrentamento dos enfermeiros em serviço hospitalar de emergência. Acta Paul Enferm. 2015; 28(3): 216-23. http://dx.doi.org/10.1590/1982-0194201500037

9. Moher D, Liberati A, Tetzlaff J, Altman DG, Group P. Preferred reporting items for systematic reviews and meta-analyses: the PRISMA statement. J Clin Epidemiol. 2009; 62(10): 1006-12.

https://doi.org/10.1016/j.jclinepi.2009.06.005

10. Santos CMDC, Pimenta CADM, Nobre MRC. The PICO strategy for the research question construction and evidence search. Rev Latino-Am Enferm. 2007; 15: 50811. http://dx.doi.org/10.1590/S0104-11692007000300023

11. Carlotto RC, Teixeira MAP, Dias ACG. Adaptação acadêmica e Coping em estudantes universitários. PsicoUSF. 2015; 20(3): 421-32. http://dx.doi.org/10.1590/1413-82712015200305

12. Kohlsdorf M. Coping strategies adopted by patients with chronic kidney disease in preparation for transplant. Psychology, Community \& Health. 2015; 4(1): 27-38. https://doi.org/10.5964/pch.v4i1.103

13. Wesner AC, Gomes JB, Detzel J, Guimarães JSP, Heldt E. Booster sessions after cognitive-behavioral group therapy for panic disorder: Impact on resilience, coping and quality of life. Behavioral and Cognitive Psychotherapy. 2015; 43: 513-25. https://doi.org/10.1017/S1352465814000289

14. Sorato D, Osório F. Coping, psychopathology, and quality of life in câncer patients under palliative care. Palliative and Supportive Care. 2015; 13: 517-25. https://doi.org/10.1017/S1478951514000339

15. Wesner AC, Gomes JB, Detzel T, Blaya C, Manfro GG, Heldt E. Effect of cognitive-behavioral group therapy for panic disorder in changing coping strategies. Comprehensive Psychiatry. 2014; 55: 87-92. http://dx.doi.org/10.1016/j.comppsych.2013.06.008 
16. Faisal-Cury A, Savoia MG, Menezes PR. Coping style and Depressive Symptomatology during Pregnancy in a Private Setting Sample. The Spanish Journal of Psychology. 2012; 15(1): 295-305. https://doi.org/10.5209/rev_SJOP.2012.v15.n1.37336

17. Negromonte MRO, Araújo TCF. Impacto do manejo clínico da dor: avaliação de estresse e enfrentamento entre profissionais de saúde. Rev Latino-AM Enfermagem. 2011;1 9(2): 7. http://dx.doi.org/10.1590/S0104-11692011000200003

18. Araújo RB, Oliveira MS, Pedroso RS, Castro MGT. Coping strategies for craving management in nicotine dependent patients. Rev. Bras. Psiquiatri. 2009; 31(2): 8994. http://dx.doi.org/10.1590/S1516-44462009000200002

19. Viana SO, Sampaio RF, Mancini MC, Parreira VF, Drummond AS. Life Satisfaction of Workers with Workrelated Musculoskeletal Disorders in Brazil: Association with Symptoms, Functional Limitation and Coping. $J$ Occup Rehabil. 2007; 17: 33-46.

http://dx.doi.org/10.1007/s10926-006-9062-z

20. Savoia MG, Bernik M. Adverse Life events and Coping Skills in Panic Disorder. Rev Hosp Clin. Fac. Med S. Paulo. 2004; 59(6): 337-40. http://dx.doi.org/10.1590/S0041-87812004000600005

21. Bassols AMS, Carneiro BB, Guimarães GC, Okabayashi LMS, Carvalho FG, Silva AB, et al. Stress and coping in a sample of medical students in Brazil. Arch Clin Psychiatry. 2015; 42(1):1-5. http://dx.doi.org/10.1590/0101-60830000000038

22. Streiner DL. Being inconsistent about consistency: when coefficient alpha does and doesn't matter. Journal of Personality Assessment. 2003; 80: 217-22. https://doi.org/10.1207/S15327752jPA8003 01

23. Bautista LM, Arias MF, Carreño ZO. Percepción de los familiares de pacientes críticos hospitalizados respecto a la comunicación y apoyo emocional. Rev Cuid. 2016; 7(2): 1297-1309. http://dx.doi.org/10.15649/cuidarte.v7i2.330

24. Alves D, Silva L, Delmondes G, Lemos IC, Kerntopf MR, Albuquerque G. Cuidador de criança com câncer: religiosidade e espiritualidade como mecanismos de enfrentamento. Rev Cuid. 2016; 7(2): 1318-24. http://dx.doi.org/10.15649/cuidarte.v7i2.336

25. Streiner DL. Being inconsistent about consistency: when coefficient alpha does and doesn't matter. Journal of Personality Assessment. 2003; 80: 217-22. https://doi.org/10.1207/S15327752JPA8003 01

26. Seidl EMF, Troccoli BT, Zannon CML. Análise Fatorial de Uma Medida de Estratégias de Enfrentamento. Psicologia: Teoria e Pesquisa. 2001; 17 (3): 225-34. http://dx.doi.org/10.1590/S0102-37722001000300004

27. Folkman S, Lazarus RS. If it changes it must be a process: A study of emotion and coping during three stages of a college examination. Journal of Personality and Social Psychology. 1985; 48: 150-70. http://dx.doi.org/10.1037/0022-3514.48.1.150
28. Gimenes MGG. A teoria do enfrentamento e suas implicações para sucessos e insucessos em psiconcologia. 1997. Em Gimenes MGG, Fávero MH. A mulher e o câncer. Campinas: Editorial Psy. 1997; (1): 111-47.

29. Savóia MG, Santana PR, Mejias NP. Adaptação do Inventário de Estratégias de Coping de Folkman e Lazarus para o português. Psicologia USP. 1996; 7 (1; 2): 183201.

30. Folkman S, Lazarus RS, Gruen RJ, De Longis A. Appraisal, coping, health status and psychological symptoms. Journal of Personality and Social Psychology. 1966; 50: 571-9. http://dx.doi.org/10.1037/0022-3514.50.3.571

31. Sánchez RT, Molina EM, Gómez-Ortega OR. Intervenciones de enfermería para disminuir la sobrecarga en cuidadores: un estudio piloto. Rev Cuid. 2016; 7(1): 1171-84. http://dx.doi.org/10.15649/cuidarte.v7i1.251 